\title{
Cuidados de enfemería en una madre de lactante con pie bot bilateral: fomentando el bienestar psicoespiritual
}

\author{
Martha Obregón-De la Torre ${ }^{1}$ \\ Amabilia Arriaga-García ${ }^{1}$ \\ Kattia Morales-Quiroz ${ }^{1}$
}

RESUMEN: El impacto de la noticia de tener un hijo con alguna malformación o discapacidad puede desencadenar en las madres problemas que afectan tanto su salud psicológica como espiritual. Estos problemas aumentan cuando los padres desconocen la enfermedad o no tienen los recursos necesarios para sobrellevar esta situación. El presente reporte de caso se trata de una madre de 40 años de edad que sufre y manifiesta vergüenza, ya que su hija presenta pie bot bilateral, acondroplasia y paladar hendido. Se aplicó el modelo de los 13 dominios para la valoración según los aspectos positivos y negativos de la taxonomía NANDA, en la cual se prioriza cuatro diagnósticos: Sufrimiento espiritual, Impotencia, Afrontamiento ineficaz y Conocimientos deficientes. Teniendo en cuenta los diagnósticos se decidió hacer un plan de cuidados de enfermería fomentando el bienestar psicoespiritual. Al finalizar el proceso se observó una ligera mejoría en el bienestar psicoespiritual. Se debe tomar en cuenta que para mejorar estos aspectos de la madre se necesitan de más intervenciones y acompañamientos e incluso de una derivación a especialista correspondiente. Se destaca que el enfermero es clave para identificar estos aspectos en las personas, ya que muchas veces es el primer contacto con las mismas en un establecimiento de salud. Con el reporte se evidencia que su labor también implica fomentar el bienestar psicoespiritual.

PALABRAS CLAVE: Pie equinovaro; Atención de enfermería; Bienestar; Espiritual.

Citar como: De la Torre M, Arriaga A, Morales K. Cuidados de enfermería en una madre de hija con pie bot bilateral: fomentando el bienestar psicoespiritual. CASUS. 2017;2(3):209-218. 


\section{INTRODUCCIÓN}

El pie bot (PB) deformidad en equinovaro o pie zambo congénito son los nombres con que se le conoce a una malformación congénita que compromete a casi todos los huesos del pie cuya causa exacta se desconoce (1). Se estima que anualmente nacen unos 120.000 niños con esta malformación y se presenta de 1 a 2 niños por 1000 nacidos vivos (1). El $80 \%$ de estos casos ocurre en países en desarrollo y por la falta de conocimiento o bajos recursos económicos la mayoría queda sin tratamiento (2). Una característica principal del PB es el pie invertido hacia adentro y se dice PB bilateral cuando se afectan ambos pies. Actualmente se está tratando de corregir con métodos no invasivos, siendo el método Ponseti una de las técnicas más importantes. Sin embargo, a comparación con otros defectos congénitos del sistema músculo-esquelético, el PB no tratado es la causa mayor de incapacidad física y laboral (2).

La asimilación del nacimiento de un hijo con un trastorno congénito como es el caso del pie bot bilateral puede ser compleja para los padres. Las expectativas que tenían con la llegada de su hijo son sustituidas por otras necesidades donde la prioridad solo será el tiempo empleado en la recuperación de su hijo (3). Adoptar la noticia de tener un hijo con alguna discapacidad afecta las relaciones intrafamiliares cambiando en las actividades diarias. En la relación de pareja se crean nuevas actitudes y comportamientos generalmente negativos. Por otro lado, teniendo en cuenta el rol de los padres en la actualidad, la mujer es la que estará más involucrada en la vida de sus hijos (3-4).

Para las madres representa una sensación de salto al vacío porque su bebé no es sano. Asimismo, experimentan diferentes emociones como por ejemplo miedo, consternación y hasta enojo (4). Esta situación por la que atraviesan estas madres no es tomada muchas veces en cuenta por los profesionales de salud (4). Por ende, al no sentirse apoyadas o atendidas pueden presentar problemas psicológicos y como consecuencia rechazan o abandonan a sus propios hijos (4).

De acuerdo a la problemática mencionada y a la poca evidencia científica sobre la temática, en el presente reporte de caso se propuso realizar intervenciones de enfermería a una madre que afronta problemas psico espirituales por tener una hija con pie bot, entre otras patologías. Estos problemas se originan a partir de que la madre no sabía cómo afrontar la situación, desconocía la patología, sufría y manifestaba tener vergüenza de contarles a sus amigos sobre la realidad de su pequeña hija. Ante la valoración del caso se realizaron cuidados holísticos priorizando el aspecto psicoespiritual. Se llevó a cabo un plan de cuidados utilizando el modelo 13 dominios de la taxonomía de clasificación de los diagnósticos North american nursing diagnosis association (NANDA) (5), Nursing Outcomes classification (NOC) (6) y Nursing interventions classification (NIC) (7). La utilización del modelo de los 13 dominios de Marjory Gordon es un sistema ampliamente utilizado en la valoración de enfermería que refleja el carácter global de cada paciente incluyendo aspectos emocionales, espirituales, de relaciones y cognoscitivos.

\section{PRESENTACIÓN DEL CASO}

Se presenta el caso de una señora de 40 años de edad, madre de una lactante menor de sexo femenino con 50 días de vida. Madre tercigesta, conviviente, con nivel de educación secundaria completa, cuenta con seguro integral de salud (SIS) y con una condición económica baja. La misma refirió haber asistido a solo nueve controles prenatales. En el séptimo mes de embarazo le indicaron que su bebé tenía malformaciones congénitas y por ello le programaron cesárea. La menor nació pretérmino pesando 3085 gr con $33 \mathrm{~cm}$ de talla. Se le diagnosticó pie bot bilateral, acondroplasia, fisura palatina, sepsis neonatal y síndrome de distrés respiratorio, por lo cual fue hospitalizada en la unidad de cuidados intensivos neonatales de un hospital público de Lima Norte.

\section{VALORACIÓN GENERAL}

Para el estudio del caso clínico se realizó la valoración mediante los 13 dominios de taxonomía NANDA, enfocando los juicios clínicos de los aspectos negativos reales a nivel holístico de la madre de la lactante con pie bot hospitalizada en el servicio de pediatría de un hospital público. Entre los aspectos negativos se tomaron en cuenta el sufrimiento, vergüenza, impotencia, desconocimiento de la madre sobre el tratamiento y evolución de la malformación congénita de su bebé (para la valoración completa ver tabla 1). 
Tabla 1. Análisis de los Dominios, según aspectos positivos y negativos

\begin{tabular}{|c|c|c|}
\hline DOMINIO & ASPECTO POSITIVO & ASPECTO NEGATIVO \\
\hline 1. Promoción de la salud. & $\begin{array}{l}\text { Asistió a nueve controles } \\
\text { prenatales. }\end{array}$ & $\begin{array}{l}\text { Gestante de } 40 \text { años de edad llega por } \\
\text { programación de cesárea. } \\
\text { RNPT AEG nació mediante cesárea con } 36 \\
\text { semanas de gestación (según test de } \\
\text { Capurro). } \\
\text { Madre manifiesta tener poca información } \\
\text { sobre el cuidado adecuado de la bebé. }\end{array}$ \\
\hline 2. Nutrición. & Peso normal. & No se presenta. \\
\hline 3. Eliminación e intercambio. & $\begin{array}{l}\text { No presenta problemas en la } \\
\text { eliminación e intercambio. }\end{array}$ & No presenta. \\
\hline 4. Actividad/Reposo. & No se observa. & $\begin{array}{l}\text { Mamá manifiesta a veces me siento } \\
\text { agotada, porque tengo que venir cada día al } \\
\text { hospital. }\end{array}$ \\
\hline 5. Percepción/Cognición. & $\begin{array}{l}\text { Madre espera adquirir } \\
\text { conocimientos sobre el cuidado } \\
\text { de la bebé. }\end{array}$ & $\begin{array}{l}\text { Madre manifiesta desconocer la patología, el } \\
\text { proceso de recuperación de su bebé y cómo } \\
\text { cuidarlo cuando salga de alta. }\end{array}$ \\
\hline 6. Autopercepción. & No se observa. & $\begin{array}{l}\text { Madre manifiesta que al inicio no tenía } \\
\text { esperanzas de que su hija se recupere. }\end{array}$ \\
\hline 7. Rol/Relaciones. & No se observa. & $\begin{array}{l}\text { Madre siente vergüenza de contarles a sus } \\
\text { amigas sobre la situación de su bebé. } \\
\text { Interrupción del contacto madre e hija. }\end{array}$ \\
\hline 8. Sexualidad. & Madre tercigesta. & No se observa. \\
\hline $\begin{array}{l}\text { 9. Afrontamiento/ Tolerancia al } \\
\text { estrés. }\end{array}$ & No se observa. & $\begin{array}{l}\text { Madre comenta que la situación le genera } \\
\text { estrés. Nunca pensó que pasaría esto con } \\
\text { su bebé. }\end{array}$ \\
\hline 10. Principios Vitales. & No se observa. & $\begin{array}{l}\text { Madre explica que le genera sufrimiento la } \\
\text { realidad que afronta. }\end{array}$ \\
\hline 11. Seguridad/Protección. & No se observa. & No se observa. \\
\hline 12.Confort & No se observa. & No se observa. \\
\hline 13.Crecimiento/Desarrollo & No se observa. & No observa. \\
\hline
\end{tabular}

\section{FASES DIAGNÓSTICA}

Según la valoración realizada a la madre se formularon diagnósticos reales utilizando la taxonomía NANDA. Se priorizaron los juicios clínicos de acuerdo a la situación que afrontaba la madre con la finalidad de brindar cuidados de enfermería para mejorar su bienestar psicoespiritual.

\section{Reales}

- (00067) Sufrimiento espiritual $\mathrm{r} / \mathrm{c}$ nacimiento de una niña con malformación congénita e/p percepción de sufrimiento. Dominio 10, Clase 3. 
- (00125) Impotencia $\mathrm{r} / \mathrm{c}$ interacciones interpersonales y sociales insuficientes $\mathrm{e} / \mathrm{p}$ vergüenza. Dominio 9, Clase 2.

- (00069) Afrontamiento ineficaz $\mathrm{r} / \mathrm{c}$ crisis situacional e/p incapacidad para manejar la situación. Dominio 9, Clase 2.

- (00126) Conocimientos deficientes r/c información insuficiente sobre la patología del bebé e/p verbalización de su madre. Dominio 5, Clase 4.

\section{FASE DE PLANIFICACIÓN}

Después de conocer la realidad que afronta la señora debido a la situación de su pequeña hija se realizó el plan de cuidados fijando espacios para hablar con ella y brindarle asesoramiento sobre los cuidados de su bebé. Se otorgó apoyo emocional con el objetivo de que desempeñe su rol y afronte la situación con herramientas necesarias para sobreponerse a esta situación.

\section{FASE DE EJECUCIÓN}

Después de la elaboración de los diagnósticos y planificación de enfermería según la valoración realizada. Se visitó a la madre para realizar un acompañamiento de soporte emocional y espiritual. Además de proporcionar información adecuada sobre la malformación de su hija los tratamientos y cuidados que debe llevar a cabo. Se ejecutó el plan de cuidados en tres visitas en el servicio donde se encontraba hospitalizada su hija.

\section{FASE DE EVALUACIÓN}

Tal como se evidencia en la tabla 2 hubo muy poca mejoría en el diagnóstico sufrimiento espiritual. Solo se consiguió recuperar una puntuación de 2 a 3 , según escala de Likert. Cabe resaltar que se le brindó apoyo espiritual para mejorar la esperanza y el optimismo de la madre mediante una comunicación terapéutica enfermera-madre para establecer confianza y una asistencia empática.

En cuanto al diagnóstico de impotencia la madre refiere "no sé qué hacer ante esta situación, me da vergüenza contarle a mis amigos lo que le pasa a mi bebé". Así mismo, se le observa cansada, sin ganas de seguir yendo a visitar a su hija al hospital, ya que manifiesta "no tengo tiempo porque tengo que trabajar". Para ello se aplicó el NIC apoyo emocional en la cual la madre pudo expresar sus emociones de ira y tristeza. Por otro lado, se aplicó el NIC dar esperanza para mejorar el NOC resiliencia personal. Se obtuvo un Likert de 1 a 2. Se logró recuperar el bienestar psicoespiritual con todas las herramientas de conocimiento y acompañamiento.

A la madre se le diagnosticó afrontamiento ineficaz ya que refiere tener vergüenza, no contar con una adecuada economía y demostró un nivel alto de estrés frente a la situación. Para ello se aplicó la intervención de enfermería para mejorar el afrontamiento mediante diferentes actividades como alentar una actitud de esperanza realista y así manejar los sentimientos de impotencia. Se obtuvo como resultado final de una puntuación de Likert de 1 a 2.

Respecto al diagnóstico de conocimiento deficiente se consiguió brindar información suficiente a la madre sobre la patología de la menor y donde podrá acudir para un tratamiento futuro, ya que ella manifestaba desconocer el diagnóstico y la evolución. Se logró una puntuación de Likert 2 a 4.

\section{DISCUSIÓN}

En el plan de cuidados a la madre se pudo observar sufrimiento, insatisfacción y cansancio debidos a la enfermedad a su hija. Igualmente le preocupaba tener que modificar su estilo de vida por no tener suficiente sustento económico. Asimismo, se le explicó el tratamiento que podría seguir la menor y se le recomendó un establecimiento de salud que podría apoyarle en la patología de su hija.

En cuanto al diagnóstico del sufrimiento espiritual la madre logró alcanzar optimismo (8). Por ello, es importante la preparación del personal de enfermería en tratar el aspecto espiritual de las personas con el fin de ayudarles en la búsqueda del crecimiento espiritual $\mathrm{y}$ de una esperanza que de sentido a sus vidas ante situaciones difíciles (8). Para ello deben tener elementos para poder intervenir en el proceso de adaptación por medio del apoyo espiritual activo para ayudar a los padres, en especial a las madres a afrontar el impacto que les produce al ver a su hijo con discapacidad, puesto que el rol del enfermero consiste en acompañar, apoyar y asesorar continuamente desde la etapa prenatal (8).

Respecto a la impotencia la madre logró adaptarse en función positiva después de un periodo de adversidad $y$ crisis. 
Tabla 2. Diagnóstico de enfermería y complicaciones potenciales: objetivos, intervenciones y resultado

\begin{tabular}{|c|c|c|c|c|}
\hline Diagnóstico de enfermería & Objetivos (NOC 1) & $\begin{array}{l}\text { Intervenciones de } \\
\text { enfermería (NIC) }\end{array}$ & Actividades & $\begin{array}{l}\text { Resultados de la } \\
\text { enfermería (NOC 2) }\end{array}$ \\
\hline $\begin{array}{l}00067 \text { Sufrimiento espiritual } \\
\text { r/c nacimiento de una niña con } \\
\text { malformación congénita } \\
\text { e/p percepción de sufrimiento. } \\
\text { Dominio 10: Principios vitales. } \\
\text { Clase 3: Congruencia entre } \\
\text { valores/ creencias/ acciones }\end{array}$ & $\begin{array}{l}1201 \\
\text { Esperanza. } \\
\text { Madre mantendrá el } \\
\text { optimismo satisfactorio y } \\
\text { revitalizante. } \\
\text { Likert: } 2 \\
\text { (Raramente demostrado) }\end{array}$ & $\begin{array}{l}5420 \\
\text { Apoyo espiritual. }\end{array}$ & $\begin{array}{l}\text { - Utilizar la comunicación } \\
\text { terapéutica para establecer } \\
\text { confianza y una asistencia } \\
\text { empática. } \\
\text { - } \text { Tratar al individuo con } \\
\text { dignidad y respeto. } \\
\text { - Compartir las propias } \\
\text { creencias sobre significado y } \\
\text { propósito según corresponda. } \\
\text { - Compartir la propia } \\
\text { perspectiva espiritual según } \\
\text { corresponda. a un } \\
\text { - Alentar la asistencia a un } \\
\text { servicio religioso si se desea. } \\
\text { - Fomentar el uso de recursos. } \\
\text { - Facilitar el uso de la } \\
\text { meditación, oración y demás } \\
\text { tradiciones. } \\
\text { Estar dispuesto a escuchar los } \\
\text { sentimientos del individuo. }\end{array}$ & $\begin{array}{l}\text { Madre mejoró el } \\
\text { optimismo satisfactorio y } \\
\text { estabilizante. } \\
\text { Indicadores: } \\
120101 \text { expresiones de } \\
\text { una orientación futura } \\
\text { positiva. } \\
120105 \text { expresiones de } \\
\text { significado de la vida. } \\
\text { 120106 expresiones de } \\
\text { optimismo. } \\
\text { 120110 expresiones de } \\
\text { sensación de } \\
\text { autocontrol. } \\
\text { Likert: } 3 \\
\text { (a veces demostrado) }\end{array}$ \\
\hline $\begin{array}{l}00125 \text { Impotencia r/c } \\
\text { interacciones interpersonales y } \\
\text { sociales insuficientes e/p } \\
\text { vergüenza. } \\
\text { Dominio 9: } \\
\text { afrontamiento/tolerancia al estrés. }\end{array}$ & $\begin{array}{l}1309 \text { Resiliencia personal } \\
\text { Madre logrará adaptación y } \\
\text { función positiva después de } \\
\text { un periodo de adversidad y } \\
\text { crisis. }\end{array}$ & 5270 Apoyo emocional & $\begin{array}{l}\text { - Realizar afirmaciones } \\
\text { empáticas y de apoyo } \\
\text { emocional con la madre. } \\
\text { - Animar a la madre a que } \\
\text { exprese sentimientos tales } \\
\text { como ansiedad, ira o tristeza y } \\
\text { ayudar a la superación. }\end{array}$ & $\begin{array}{l}\text { Madre logra } \\
\text { progresivamente la } \\
\text { adaptación y función } \\
\text { positiva después de la } \\
\text { adversidad y crisis. }\end{array}$ \\
\hline
\end{tabular}




\section{Tabla 2. continúa}

Clase 2: respuestas de

afrontamiento.
Likert: 1

(nunca demostrado)
5310 Dar esperanza
- Comentar con la madre experiencia emocional.

- Comentar las consecuencia de no abordar los sentimientos de culpa vergüenza.

- Remitir a servicios de asesoramiento, o grupos de apoyo si se precisa.

Indicadores:

130901 verbaliza una actitud positiva.

130902 utiliza estrategias de afrontamiento efectivas.

130906 muestra un estado de ánimo positivo.

130912 busca apoyo emocional

Likert: 2 (algunas veces demostrado)

00069
Afrontamiento ineficaz $\mathrm{r} / \mathrm{c}$ crisis
situacional e/p incapacidad para manejar la situación.

1504 soporte social

Madre obtendrá ayuda fiable para afrontar con eficacia la

situación.

Likert: 1

(Inadecuado)

Dominio

9:afrontamiento/tolerancia al

estrés

Clase 2:respuestas de

afrontamiento

5230 Mejorar el

afrontamiento
- Alentar una actitud de Madre obtiene ayuda fiable esperanza realista como para afrontar con eficacia la forma de manejar los situación. sentimientos de impotencia.

- Ayudar a la paciente a Indicadores: desarrollar una valoración objetiva del acontecimient Estudiar con la paciente para buscar ayuda en otras métodos correctos de manejo personas. de situaciones difíciles.

- Presentar a la paciente personas que hayan pasado por la misma experiencia y superaron con éxito.

150404 refiere información
150412 refiere ayuda ofrecida por los demás. proporcionada por otras personas. 


\section{Tabla 2. continúa}

150410 refiere contactos

sociales de soporte

adecuados

Likert: 2

(ligeramente inadecuado)

\section{6}

Conocimiento deficiente $r / c$

información insuficiente sobre la

patología del bebé/p verbalización

de su madre.

Dominio 5:percepción/cognición

clase 4:cognición
1803 Conocimiento:

proceso de la enfermedad

6820 Cuidados de

lactante

Madre obtendrá

conocimientos sobre el

proceso de la enfermedad de

la bebé y las complicaciones

potenciales

Likert: 2

(conocimiento escaso)

\section{Asesoramiento} que realicen los cuidados especiales de la lactante.

- Proporcionar información a la

madre.

- Proporcionar información Indicadores:

objetiva según sea necesario 180303 causas o factores y según corresponda.

- Favorecer la expresión asertiva de emociones sentimientos.

\section{contribuyentes.}

180306 signos y síntomas de la enfermedad.

180309 complicaciones potenciales de la enfermedad.

180317 fuentes acreditadas de información sobre la

enfermedad específica.

Likert: 4

(conocimiento sustancial). 
Se pudo percibir que aún no ha disminuido considerablemente la vergüenza que siente. Los padres tienen que enfrentar una experiencia nueva ante la presencia de un hijo(a) con esta malformación por lo que tendrán que desarrollar, de forma progresiva, habilidades especiales de cuidado (9). Asimismo, es necesario recordarles que pueden disfrutar al lado de su hijo de la misma manera que cualquier otro padre lo hace, demostrando afecto, observando su crecimiento y desarrollo, no obstante las diferencias. Del mismo modo, pueden compartir la felicidad con los miembros de su familia y amigos (9).

Frente al diagnóstico afrontamiento ineficaz la madre logró obtener ayuda fiable para afrontar con eficacia la situación del problema que presenta su bebé. La presencia de un hijo con malformaciones congénitas constituye para la familia un factor de perturbación tanto psicológico, como social y económico. Para muchas familias no es fácil afrontar esta situación ya que representa una mayor atención y dedicación. Igualmente supone superar los sentimientos de culpabilidad y/o rechazo que en algunas ocasiones sufren (10). Por lo tanto, para restaurar el equilibrio emocional y la adaptación frente a la nueva realidad es esencial que la madre cambie la percepción respecto a su hijo. Del mismo modo, es importante que la madre exprese sus emociones para que disminuya sus cargas e identificar cómo se encuentra ella (11).

Respecto al conocimiento deficiente la madre logró obtener conocimientos sobre el cuidado adecuado de su hija. El pie de Bot es un trastorno congénito poco conocido, debido a ello se le hizo una intervención a la madre para ver cuánto sabía acerca de la patología (2). Se explicó a la madre a cerca de los cuidados que debe recibir la lactante y sobre alternativas de tratamiento y/o recuperación a través de la atención profesional de un fisioterapeuta o cirujano especializado.

Una de las dificultades que se encontró fue el factor tiempo, ya que estos aspectos psicoespirituales necesitan de varios encuentros para poder mejorar estos aspectos completamente. Igualmente, no se logró la participación de su pareja. Cabe resaltar que el enfermero es uno de los primeros contactos entre el personal de salud por lo tanto, debe identificar problemas para poder intervenir o derivar al profesional correspondiente.
Se recomienda que el personal de salud se enfoque en todos los aspectos del ser humano. Para ello debe estar capacitado en cómo intervenir cuando una persona afronta una situación como en el presente caso. Asimismo, deben crearse normas o guías sobre el cuidado holístico e integral al recién nacido con discapacidad y su familia. Finalmente, la enfermera debe realizar cuidados de enfermería pertinentes basándose en la taxonomía de NANDA, NOC y NIC que respondan a la recuperación de un bienestar integral del paciente.

\section{REFERENCIAS BIBLIOGRÁFICAS}

1. Hamilton G. Pie Zambo: El Método de Ponseti.1 ed. Estados Unidos: Global-Help Organization; 2003.

2. Masquijo J, Marchegiani S, Allende V. Diagnóstico prenatal del pie bot: Revista de la Asociación Argentina de Ortopedia y Traumatología. 2011;75(4):14-18.

3. Aldivia, M. Estudio descriptivo observacional retrospectivo en población hospitalaria con el objetivo de identificar los factores asociados a la recidiva del pie bot tratado con el método de Ponseti. [Título Médico- Cirujano]. Lima: Universidad Ricardo palma. En el hogar clínica San Juan de Dios entre los años 2009-2012.

4. Alarcon S, Whetsell $M$, Cadena S, Yañes G: Experiencias Vividas de Madres que Tienen un Hijo con Labio Leporino y Paladar Hendido. Desarrollo Cientif Enferm. 2011; 19(5): 166-170.

5. Herdman TH, Kamitsuru S. NANDA International. Diagnósticos enfermeros: Definiciones y clasificación, 2015-2017. Barcelona: Elsevier; 2015.

6. Moorhead S, Johnson M, Mass ML, Swanson E, Clasificación de Resultados de Enfermería (NOC). 5ta ed. Barcelona: Elsevier; 2014.

7. Bulecheck GM, Butcher HK, Dochterman JM, Wagner CM. Clasificación de intervenciones de Enfermería (NIC). 6ta ed. Barcelona: Elsevier; 2014.

8. Muñoz D, Morales I, Bermejo J, González J. La Enfermería y los cuidados del sufrimiento espiritual. Índex de Enfermería. 2014;23(3):1132-1296.

9. Gómez Ruiz JC, Fernández N, Páez P, Zárate I. M. Detección de Anomalías Congénitas en 12,760 
nacimientos de tres hospitales en la ciudad de Bogotá Colombia 2004-2005 mediante ecografía prenatal. Rev. Colombiana de Obstetricia y Ginecología. 2007;58:194-20.

10. Johnson $\mathrm{M}$, Bulecheck $\mathrm{G}$, Butcher $\mathrm{H}$. Interrelaciones NANDA, NOC y NIC. Diagnósticos enfermeros, resultados e intervenciones. 2da ed. Barcelona: Elsevier; 2007.

11. Escobar A, Hernández L, Morales V. Beliefs and practices of caring for pregnant women with diagnosis of fetal malformation. Investigación y Educación en Enfermería. 2013;(2):234-242. 
ISSN 2519-0652 CASUS.2017;2(3):209-218

\section{Nursing care on infant's mother with bilateral bot foot: fostering psychospiritual well-being}

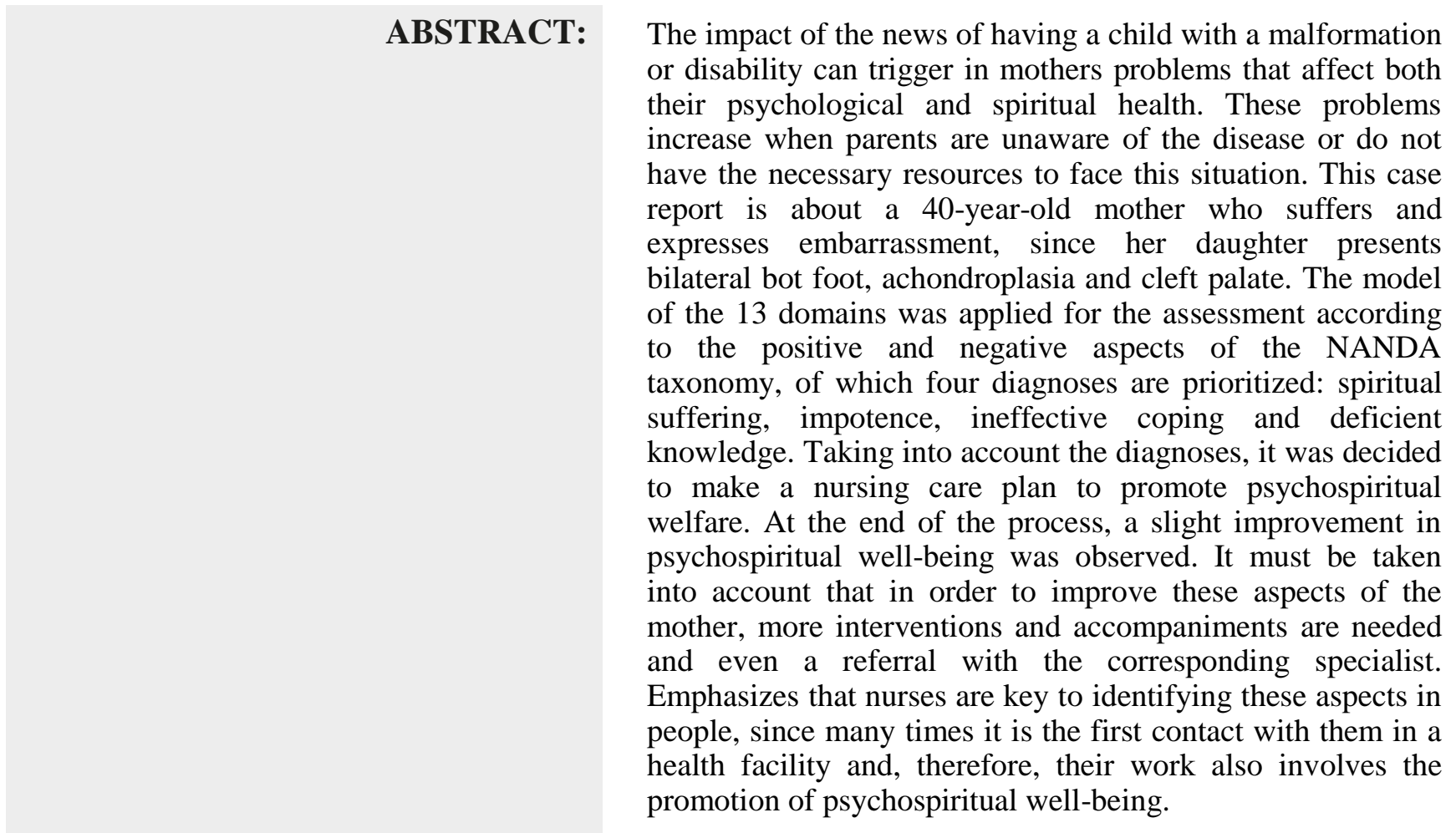

KEY WORDS: $\quad$ Clubfoot; Nursing care; Wellness; Spiritual. 